\title{
Análisis de la arquitectura empresarial como oportunidad de mejora en las microempresas de la ciudad de Cuenca
}

\section{Analysis of business architecture as an opportunity for improvement in microenterprises in the city of Cuenca}

\section{Análise da arquitetura de negócios como oportunidade de melhoria em microempresas na cidade de Cuenca}

Fausto Germán Palacios-Urgilés I

faustogpu@gmail.com

Milton Alfredo Campoverde-Molina II

mcampoverde@ucacue.edu.ec

*Recibido: 19 de mayo de 2019 *Aceptado: 10 de junio de 2019 * Publicado: 05 de julio de 2019

I Ingeniero de Sistemas, Jefe de Sistemas Indumot S.A., Universidad Católica de Cuenca, Cuenca, Ecuador.

II Magíster en Docencia Universitaria, Magíster en Evaluación y Auditoria de Sistemas Tecnológicos, Ingeniero en Sistemas, Docente de la Unidad Académica de Tecnologías de la Información, Jefatura de Posgrados, Universidad Católica de Cuenca, Cuenca, Ecuador. 


\title{
Resumen
}

La acelerada evolución tecnológica, así como el constante cambio económico, han provocado que las microempresas tengan que buscar la manera de adaptarse y afrontar dichos cambios, no como una dificultad sino como una oportunidad de desarrollo. Si una microempresa no puede adaptarse al mercado cambiante puede fracasar. Muchos estudios se han focalizado en empresas medianas y pequeñas, dejando de lado a las microempresas que son las más numerosas. Este artículo propone generar una visión preliminar de las microempresas. $\mathrm{Y}$ en base a esta visión preliminar determinar cuál es el principal problema común para las microempresas de Cuenca. Para obtener una visión de arquitectura deseada para su principal problema. Para lograr esta visión de arquitectura deseada se crea un portafolio de proyectos. El principal resultado que se obtuvo fue que el mayor problema común a las microempresas tiene que ver con la falta de una Arquitectura de Negocio. Por consiguiente, las microempresas navegan a la deriva sin un horizonte claro. Si las microempresas aplican los correctivos propuestos en esta investigación van a llegar a la visión de arquitectura deseada. Esto permitiría a las microempresas tener objetivos claros, y plantear mejoras que apoyen el negocio, haciéndolas más rentables y adaptables al cambio.

Palabras clave: Arquitectura empresarial; microempresa; TOGAF; outsourcing; proyectos.

\begin{abstract}
The accelerated technological evolution, as well as the constant economic change, have caused that the microenterprises have to look for the way to adapt and to confront these changes, not as a difficulty but as an opportunity of development. If a microenterprise cannot adapt to the changing market, it may fail. Many studies have focused on medium and small enterprises, leaving aside the microenterprises that are the most numerous. This article proposes to generate a preliminary vision of microenterprises. And on the basis of this preliminary vision, determine what is the main common problem for microenterprises in Cuenca. To obtain a vision of desired architecture for their main problem. To achieve this desired architectural vision, a portfolio of projects is created. The main result obtained was that the biggest problem common to microenterprises has to do with the lack of a Business Architecture. Consequently, microenterprises drift without a clear horizon. If the microenterprises apply the correctives proposed in this research, they will arrive at the desired vision
\end{abstract}


of architecture. This would allow microenterprises to have clear objectives, and to propose improvements that support the business, making them more profitable and adaptable to change.

Keywords: Business architecture; small business; TOGAF; outsourcing; projects.

\section{Resumo}

A acelerada evolução tecnológica, assim como a constante mudança econômica, fizeram com que as microempresas tivessem que encontrar uma maneira de se adaptar e enfrentar essas mudanças, não como uma dificuldade, mas como uma oportunidade de desenvolvimento. Se uma microempresa não pode se adaptar ao mercado em mudança, pode falhar. Muitos estudos se concentraram em empresas de médio e pequeno porte, deixando de lado as microempresas que são as mais numerosas. Este artigo propõe gerar uma visão preliminar das microempresas. E com base nessa visão preliminar, determine o principal problema comum para as microempresas em Cuenca. Para obter uma visão de arquitetura desejada para o seu problema principal. Para alcançar essa visão arquitetônica desejada, é criado um portfólio de projetos. O principal resultado obtido foi que o maior problema comum às microempresas tem a ver com a falta de uma arquitetura de negócios. Consequentemente, as microempresas derivam sem um horizonte claro. Se as microempresas aplicarem as medidas corretivas propostas nesta investigação, elas atingirão a visão arquitetônica desejada. Isso permitiria às microempresas ter

objetivos claros e propor melhorias que apoiassem os negócios, tornando-os mais lucrativos e adaptáveis à mudança.

Palavras-chave: Arquitetura empresarial, microempresa, TOGAF, terceirização, projetos.

\section{Introducción}

La acelerada evolución tecnológica, así como el constante cambio económico, han provocado que empresas tanto públicas como privadas tengan que buscar la manera de adaptarse y afrontar dichos cambios, no como una dificultad sino como una oportunidad de desarrollo. Esto ha dado paso a varios marcos o frameworks que permitan la implementación de una arquitectura empresarial para afrontar estos cambios (Erika María González Escobar, 2010). Esta necesidad ha dado como resultado que en Arquitectura Empresarial existan varios marcos de trabajo, como, por ejemplo: ZACHMAN, TOGAF, FEAF, FEA, DODAF, GEAF, EZAF, BTEP, E2AF, etc. 
En su mayoría los gerentes de las microempresas no visualizan la importancia que puede tener la tecnología en el cumplimiento de sus objetivos estratégicos. Esto se debe a que el gerente de la microempresa en América Latina generalmente es empírico y no tiene como hábitos el capacitarse y actualizarse (Camacho, 2008). De acuerdo a estudios realizados por la Fundación para el Desarrollo Sostenible en América Latina (FUNDES) las microempresas apenas invierten un 2\% de su presupuesto en tecnología. A esto sumemos que en su mayoría las microempresas no tienen contemplado un departamento de tecnología dentro de su estructura organizacional. Las microempresas en su mayoría tienen un outsourcing de Tecnologías de la Información (TI).

Hoy en día la Globalización obliga a las empresas a innovar con nuevas tendencias tecnológicas que promueva la diversificación, incremente la productividad y reduzca las brechas tecnológicas en nuestra región. Ecuador registró en el 2016 a 843.745 empresas y de estas el 90,5\% son microempresas, es decir, empresas con ventas anuales menores a 100 mil dólares y entre 1 y 9 empleados (Instituto Nacional de Estadística y Censos, 2017). Las microempresas ecuatorianas, en su mayoría, tienen una estructura empírica. Esta estructura empírica se convierte en un obstáculo para las microempresas al momento de hacer frente a los cambios de mercado. El no poder adaptarse a estos cambios da como resultado el fracaso de estos emprendimientos. En Ecuador al igual que en el resto de Latinoamérica las microempresas adolecen fallas económicas, organizacionales, tecnológicas o de conocimiento desde el momento mismo de su constitución. Estas fallas se traducen en desventajas competitivas dentro de un mercado cambiante.

De acuerdo a las resoluciones del Servicio de Rentas Internas No. NACDGERCGC13-00236, publicada en Registro Oficial No. 956 del 17 de mayo de 2013 (Servicio de Rentas Internas, 2013) y No. NAC-DGERCGC14- 00157 publicada en el suplemento del Registro Oficial No. 215 del 31 de marzo del 2014 (Servicio de Rentas Internas, 2014), informó los calendarios de obligatoriedad para el ingreso al sistema de facturación electrónica por parte de los sujetos pasivos del sector privado y sector público respectivamente. A pesar de que esta normativa no afecta a las microempresas, muchas de estas ya se encuentran en periodos de prueba de la herramienta tecnológica sin una adecuada gestión del cambio, producto de la falta de una adecuada arquitectura empresarial. 
La importancia de este trabajo radica en poder extraer una visión preliminar de los errores más comunes que cometen las microempresas, con respecto al uso de las tecnologías y a su innovación. Poder analizar dichos errores y determinar correctivos comunes que puedan servir a todas las microempresas de la ciudad de Cuenca.

El propósito de esta investigación es obtener una vista preliminar de la Arquitectura Empresarial de las microempresas. Determinar que dimensión de Arquitectura Empresarial presenta más problemas para la microempresa. Y finalmente proponer los correctivos necesarios para obtener una primera Visión de Arquitectura que permita a la microempresa ser competitiva y adaptable a los cambios del mercado. Basado en estas premisas esta investigación busca responder las siguientes preguntas:

¿Cuál es el error más común en las microempresas de la ciudad de Cuenca?

¿Cuál es el nivel tecnológico de las microempresas de la ciudad de Cuenca?

En cuanto a la estructura y contenido del artículo, en la sección 2 se presentan los conceptos relacionados con la investigación. En la sección 3, la revisión de diferentes fuentes bibliográficas de experiencias y resultados de investigaciones de aplicación de arquitectura empresarial. En la sección 4, se detalla la metodología utilizada para desarrollar esta investigación. En la sección 5, se muestran los resultados de la visión preliminar de la arquitectura empresarial presente en las microempresas de la ciudad de Cuenca. En la sección 6, se establecen las conclusiones de acuerdo a los resultados obtenidos.

\section{Desarrollo}

\section{La microempresa}

La microempresa según (Cardozo, 2004) se define como: “...una unidad productiva de pequeña escala, con un máximo de diez (10) trabajadores, con capacidad real de generar empleos, que por su flexibilidad es capaz de ajustarse a condiciones de mercado, lo que le permite mantener condiciones de productividad, registradas y con control contable, gerenciadas bajo la premisa de que la tecnología (esto es, la utilización del conocimiento) es la clave fundamental para la competitividad."

\section{El Outsourcing}


El contratar servicios, en especial en épocas de crisis, es una estrategia que utilizan las empresas para reducir costos. Los servicios brindados son amplios y se pueden contratar para cualquier área del negocio. Esto ha introducido el concepto de todo como servicio (XaaS) que no es más que brindar accesos a un producto bajo demanda. Como sabemos el proveer tecnología como servicio no es un concepto nuevo en el mundo empresarial. En los últimos años se ha incrementado exponencialmente esta modalidad y se ha expandido a una diversidad de productos dando como resultado que el XaaS esté haciendo que muchas empresas, que tradicionalmente ignoraban por completo la tecnología, hoy en día accedan a esta, en especial las Pymes. El XaaS ha permitido flexibilidad a las empresas, especialmente en América Latina, permitiendo su adaptabilidad a los diferentes escenarios económicos que puedan atravesar (Jones, n.d.).

\section{Arquitectura empresarial}

La arquitectura empresarial nos permite conectar las relaciones entre las iniciativas de negocio y la tecnología que las soporta. Dan la pauta para que cada departamento defina sus propios objetivos de manera q apuntalen los objetivos de la empresa.

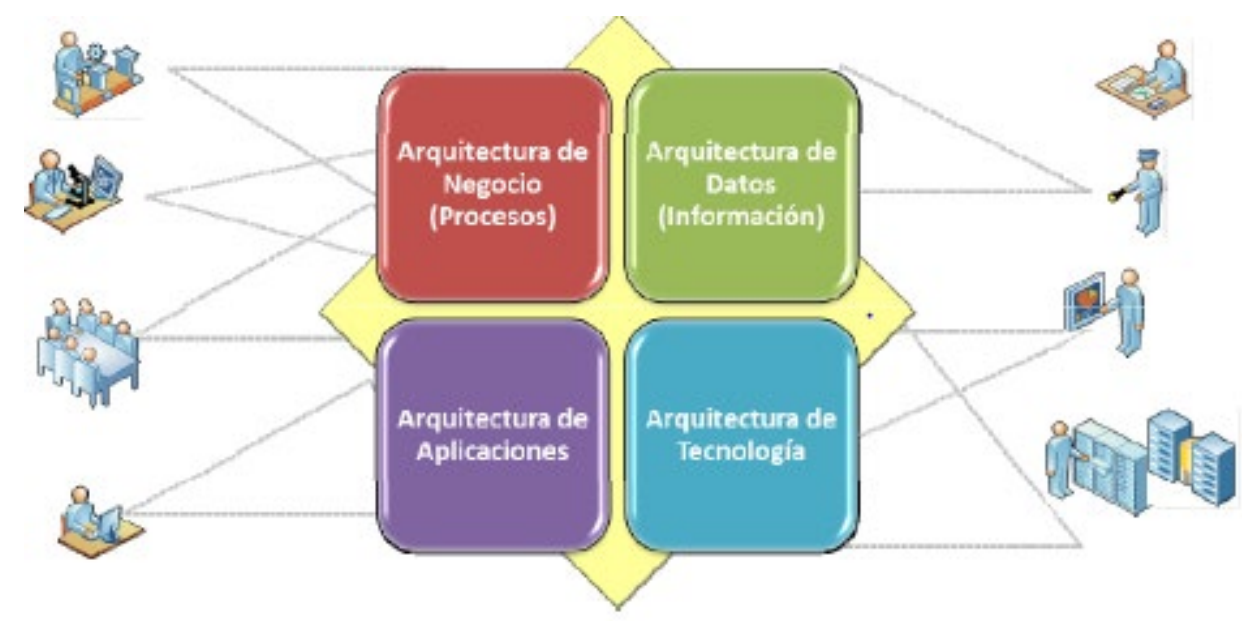

Figura 1. Arquitectura empresarial e Interrelaciones

Fuente: (Cortés, 2013) 


\section{Framework de The Open Group Architecture Framework (TOGAF)}

Es un framework de arquitectura empresarial desarrollado por el Open Group quien está dedicado a la generación de soluciones tecnológicas en el campo de las telecomunicaciones. Entre sus miembros contamos con reconocidas empresas como son: Fujitsu, Hitachi, HP y la NASA. Su primera versión fue lanzada en el año 1995 que estaba basada en TAFIM (Technical Architecture framework for Information Management).Este modelo planteaba utilizar sistemas abiertos y nuevas tecnologías que permita desarrollar una aplicación que cubriera todos los estándares de calidad, en toda su infraestructura, identificando servicios, conceptos, componentes y configuraciones (The Open Group, 2012). 
Para nuestro estudio vamos a utilizar la versión 9.1 de TOGAF ya que la versión 9.2 se lanzó el 16 de abril del 2018 y aún se dispone de información reducida. TOGAF cubre las arquitecturas que se describen en la siguiente Tabla 1.

\begin{tabular}{|c|c|}
\hline Dimensiones de Arquitecture & \\
\hline Tipo de Arquitectura & Descripción \\
\hline Arquitectura de Negocio & $\begin{array}{l}\text { La estrategia de negocio, gobierno, } \\
\text { organización y procesos clave de la } \\
\text { organización. }\end{array}$ \\
\hline Arquitectura de Datos & $\begin{array}{l}\text { La estructura de datos lógicos y } \\
\text { físicos que posee una organización y } \\
\text { sus recursos de gestión de datos. }\end{array}$ \\
\hline Arquitectura de Aplicación & $\begin{array}{l}\text { Un plano (blueprint en inglés) de las } \\
\text { aplicaciones individuales a } \\
\text { implementar, sus interacciones y sus } \\
\text { relaciones con los procesos de } \\
\text { negocio principales de la } \\
\text { organización. }\end{array}$ \\
\hline Arquitectura Tecnológica & $\begin{array}{l}\text { Incluye infraestructura de TI, capa } \\
\text { de mediación (middleware en } \\
\text { inglés), redes, comunicaciones, } \\
\text { procesamiento y estándares. }\end{array}$ \\
\hline
\end{tabular}

Tabla 1: Dimensiones de arquitectura empresarial soportados por TOGAF Fuente: (The Open Group, 2012) 
Vol. 5, núm. 3, julio 2019, pp. 487-512

Análisis de la arquitectura empresarial como oportunidad de mejora en las microempresas de la ciudad de Cuenca

TOGAF se compone de 3 herramientas:

\section{Método de desarrollo de arquitectura (ADM).}

EL Método de Desarrollo de la Arquitectura conocido como ADM por sus siglas en inglés nos definen las fases que debemos recorrer para implementar las diferentes Arquitecturas que soporta TOGAF.

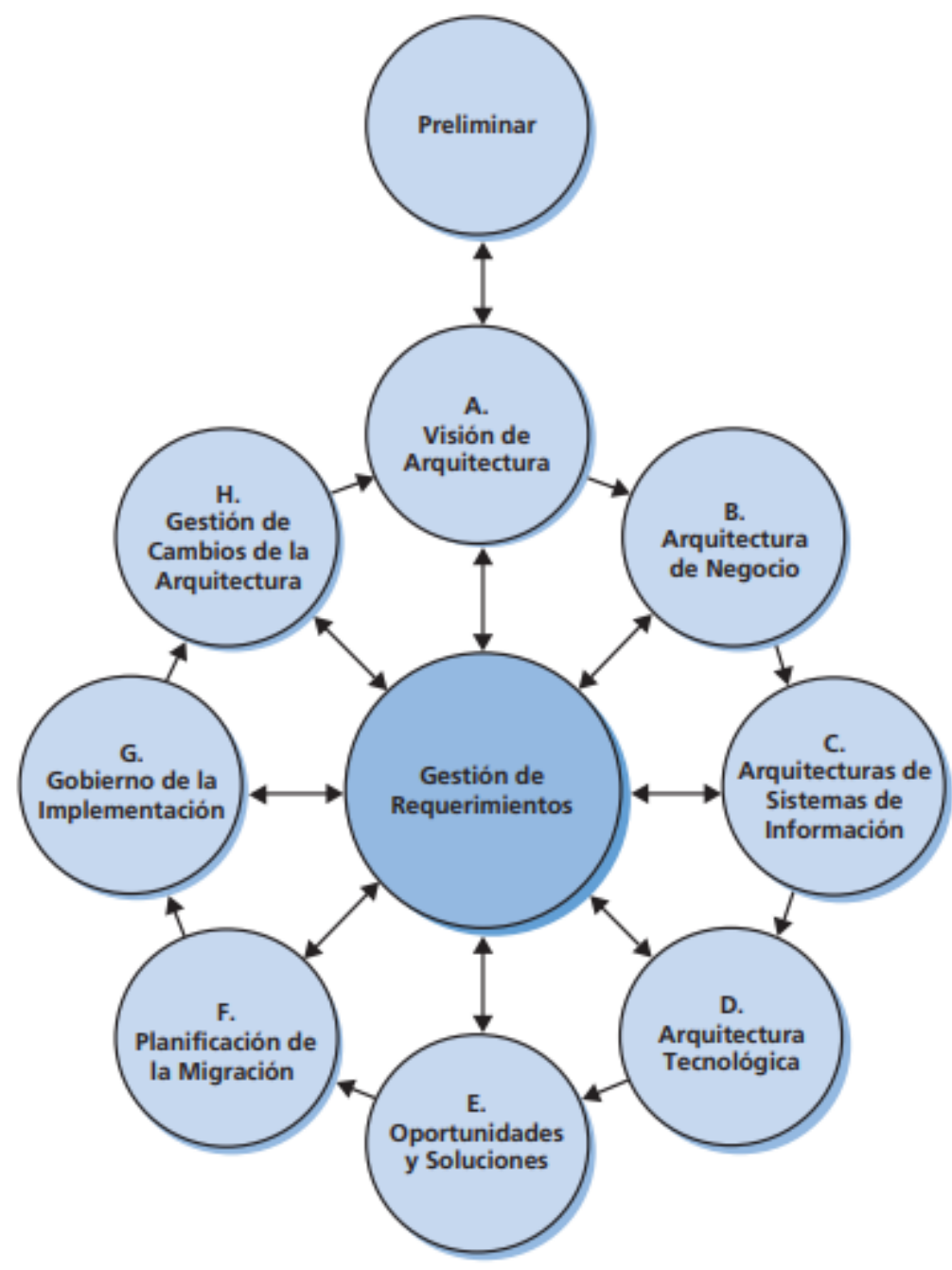

Figura 2. ADM de TOGAF

Fuente: (The Open Group, 2011) 


\begin{tabular}{|c|c|}
\hline \multicolumn{2}{|c|}{ Fases del ADM a usar en esta Investigación } \\
\hline Fase & Actividades \\
\hline Preliminar & $\begin{array}{l}\text { Prepara a la organización para la } \\
\text { implementación de la Arquitectura } \\
\text { empresarial. } \\
\text { Selección de herramientas y definición de } \\
\text { principios de Arquitectura empresarial. }\end{array}$ \\
\hline $\begin{array}{c}\text { A. } \\
\text { Vision de } \\
\text { Arquitectur }\end{array}$ & $\begin{array}{l}\text { Determina el alcance, las limitaciones y } \\
\text { las expectativas de un proyecto de } \\
\text { implementación de AE (Arquitectura } \\
\text { empresarial). } \\
\text { Valida el contexto de negocio. } \\
\text { Crea la declaración de trabajo de la AE. } \\
\text { Obtiene las aprobaciones }\end{array}$ \\
\hline
\end{tabular}

Tabla 2: Fases del ADM de TOGAF a usarse en la investigación.

Fuente: (The Open Group, 2012)

\section{Contenedor Empresarial (Continuum Empresarial)}

Es la vista del repositorio de la arquitectura, el cual nos define métodos para clasificar las arquitecturas y soluciones, mientras estén evolucionando desde arquitecturas fundamentales genéricas hasta arquitecturas específicas para una organización.

\section{Repositorio de arquitectura}

Brinda apoyo al continuum empresarial y permite almacenar los entregables creados por el ADM, con el fin de facilitar la comunicación, cooperación y entendimiento entre las diferentes áreas. 


\section{Trabajos relacionados}

Varios estudios se han realizado para identificar brechas entre lo que disponen las empresas y las mejoras que se pueden llevar a cabo mediante la aplicación de Arquitectura Empresarial. Un ejemplo de esto es el estudio realizado en donde se definieron las diferentes brechas existentes en el negocio y plantea un portafolio de proyectos para mitigar las brechas (Base et al., 2014).

Las empresas Pymes o pequeñas, según la resolución de la Comunidad Andina o CAN, se definen como empresas cuyo personal va de 10 a 49 empleados y sus ingresos oscilan entre $\$ 100.001$ y \$ 1.000.000 de dólares. Un estudio realizado nos presentó las ventajas que tiene el aplicar una Arquitectura Empresarial para la mediana empresa (Canabal, Cabarcas, \& Martelo, 2017). Y nos planteó el uso de aplicaciones colaborativas de Google como vehículo para su implementación.

Un estudio realizado en el año 2017 nos planteó utilizar un Enfoque Ágil (Sandoval, Galvez, \& Moscoso, 2017). Este estudio nos plantea implementar TOGAF utilizando un Enfoque Ágil como metodología de trabajo para los equipos de Arquitectura Empresarial. Este enfoque nos planteó una mejora en tiempos de implementación.

De acuerdo a los resultados obtenidos en las investigaciones revisadas, podemos observar que los estudios se centran en empresas grandes medianas y pequeñas. Tomando en cuenta esta premisa se plantea una investigación basada en el análisis de la Arquitectura Empresarial en las microempresas de la ciudad de Cuenca. Con esta investigación vamos a obtener una visión preliminar y llegar a determinar los errores más comunes que cometen las microempresas de la ciudad de Cuenca.

\section{Metodología}

A continuación, se describen los pasos utilizados en esta investigación

1. Selección de las microempresas: en esta investigación se realiza el análisis de las microempresas que comercializan productos de construcción. Se toma como muestra a 203 microempresas de la ciudad de Cuenca como objeto de estudio.

2. Validación y aplicación del estudio: el estudio fue validado por gerentes de desarrollo organizacional y TI de Cuenca. Se aplicó el estudio a una muestra de microempresas 
comercializadoras de productos de la construcción. En el estudio participaron gerentes y empleados de las microempresas.

3. Visión preliminar de las microempresas de la Ciudad de Cuenca: en esta sección se va a realizar un estudio comparativo de las diferentes dimensiones de Arquitectura Empresarial encontradas. Para elegir que dimensión de Arquitectura Empresarial representa el mayor problema para las microempresas.

4. Elaboración de la propuesta - Visión de Arquitectura: en esta sección se va a plantear una propuesta, que permita a las microempresas alcanzar una primera Visión de Arquitectura.

5. Portafolio de Proyectos: en esta sección vamos a definir un portafolio de proyectos que nos permitan mejorar la dimensión de Arquitectura Empresarial con mayores problemas en las microempresas.

\section{Resultados}

\section{Selección de las microempresas.}

De acuerdo a los datos que observamos en el Gráfico 1, por actividad económica la mayor parte de microempresas se dedican al comercio al por mayor y menor, le sigue la reparación de vehículos automotores y motocicletas con 308.956 (Censos, 2016). 
Dom. Cien., ISSN: 2477-8818

Vol. 5, núm. 3, julio 2019, pp. 487-512

Análisis de la arquitectura empresarial como oportunidad de mejora en las microempresas de la ciudad de Cuenca

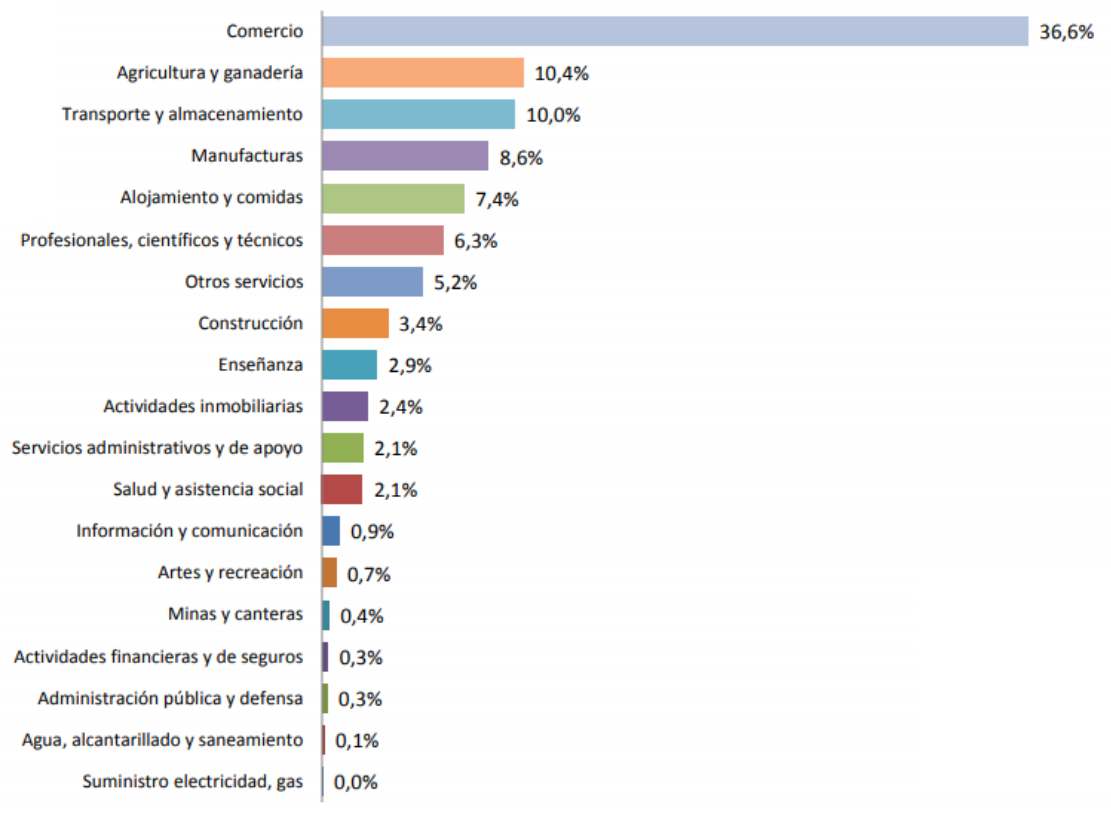

Gráfico 1. Estructura de empresas por actividad económica, año 2016.

Fuente: (Censos, 2016)

Ya que el mayor número de microempresas se encuentran concentradas en la actividad económica comercio, en esta investigación vamos a estudiar solo microempresas comercializadoras al por mayor y menor de productos para la construcción de la ciudad de Cuenca.

Para definir la muestra a la cuál aplicar la encuesta se va a utilizar la fórmula que permite calcular la muestra cuando se desconoce el tamaño de la población por falta de datos oficiales (Torres, M., Paz, K., \& Salazar, 2006), como en nuestro caso. 


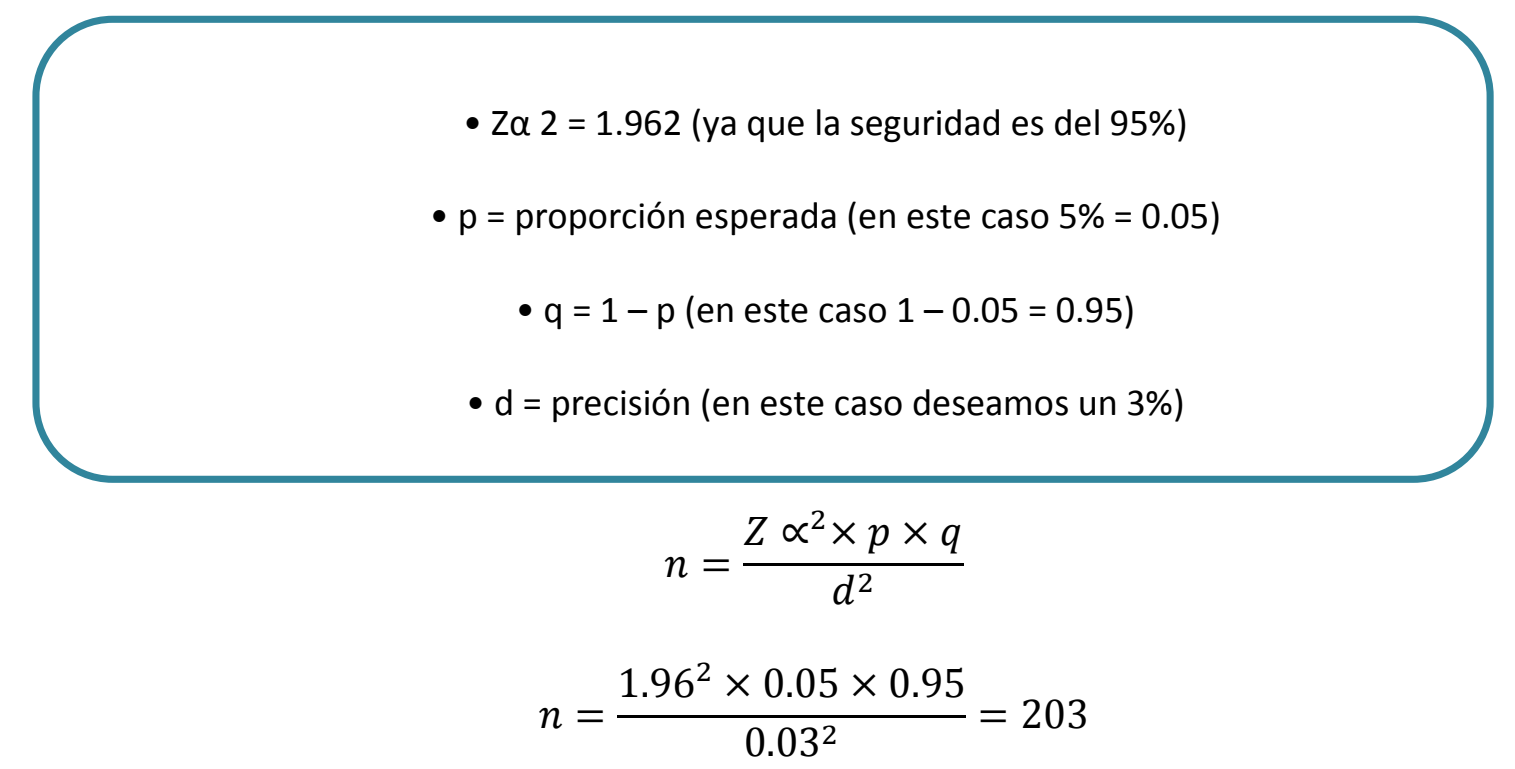

Se tiene que aplicar la encuesta a 203 microempresas comercializadoras para alcanzar un nivel de confianza del $95 \%$.

\section{Validación y aplicación del estudio.}

Para crear las encuestas se utiliza el criterio de expertos en el tema de la Arquitectura de Negocio, Datos, Aplicaciones y Tecnología.

Se formulan encuestas dirigidas a los gerentes propietarios y a empleados de las microempresas. Se utilizó el Google Forms para distribuir las encuestas. Se enviaron por correo a 500 microempresas de la ciudad de Cuenca para ser llenadas por los gerentes propietarios y por los empleados. De estas empresas apenas 192 respondieron la encuesta. Pero de estas 192 microempresas 10 respondieron que tenían más de 10 empleados, quedando eliminadas por no cumplir con el criterio de lo que es una microempresa. Para lograr completar la muestra se escogió al azar microempresas ferreteras de la ciudad de Cuenca para aplicar las encuestas de manera presencial utilizando un formulario impreso. Se escogieron 30 ferreterías en caso de que no todas puedan responder la encuesta. De estas 30 ferreterías apenas 17 dieron apertura para aplicar el formulario. Para lograr completar la muestra se escogieron 10 microempresas comercializadoras de pintura. De estas 5 nos permitieron aplicarles la encuesta logrando ajustar un total de 204 encuestas. Se procedió a eliminar una encuesta al azar para tener la muestra de 203 microempresas comercializadoras de productos para la construcción. 


\section{Visión preliminar de las microempresas comercializadoras de la Ciudad de Cuenca.}

De los resultados obtenidos de la aplicación de la encuesta a gerentes propietarios, de acuerdo a las dimensiones de Arquitectura Empresarial contempladas en TOGAF, se pudo determinar que el 100\% de las microempresas encuestadas no disponen de una arquitectura de negocio. Esto quiere decir que no poseen una misión, visión, plan estratégico, ni una metodología para la gestión de proyectos. El 96,4\% de microempresas no tienen definidos sus procesos de manera formal. En el Gráfico 2 podemos ver las dimensiones de TOGAF de acuerdo a la Arquitectura Empresarial, desde la perspectiva de los Gerentes Propietarios.

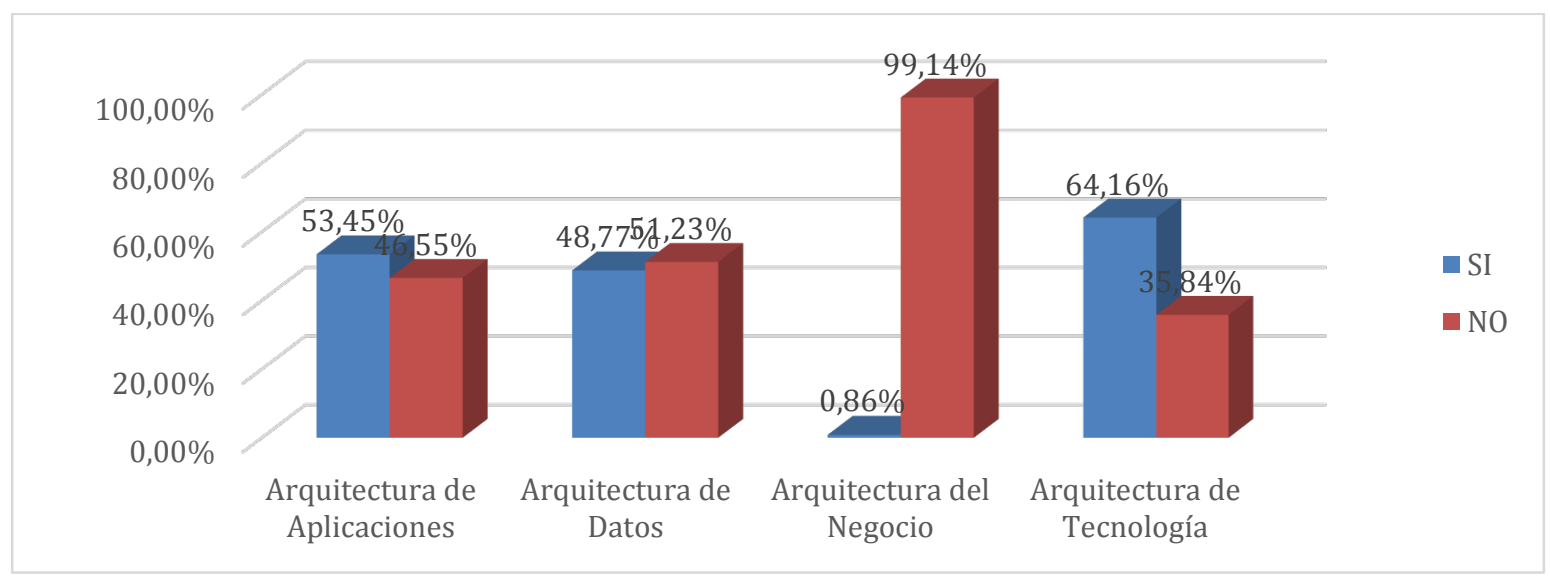

Gráfico 2. Dimensiones de TOGAF, desde la vista del Gerente Propietario.

Fuente: Elaboración propia

Como pudimos observar las microempresas tienen problema en todas las dimensiones de Arquitectura Empresarial desde el punto de vista de Gerentes Propietarios. Pero de todas las dimensiones la Arquitectura de Negocio es la que mayores problemas representa. Algo interesante que se observa es que la Arquitectura Tecnológica desde el punto de vista de Gerentes Propietarios es una de las dimensiones que mejor resultado obtuvo. En el Gráfico 3 podemos ver las dimensiones de TOGAF de acuerdo a la Arquitectura Empresarial, desde la perspectiva de los empleados. 


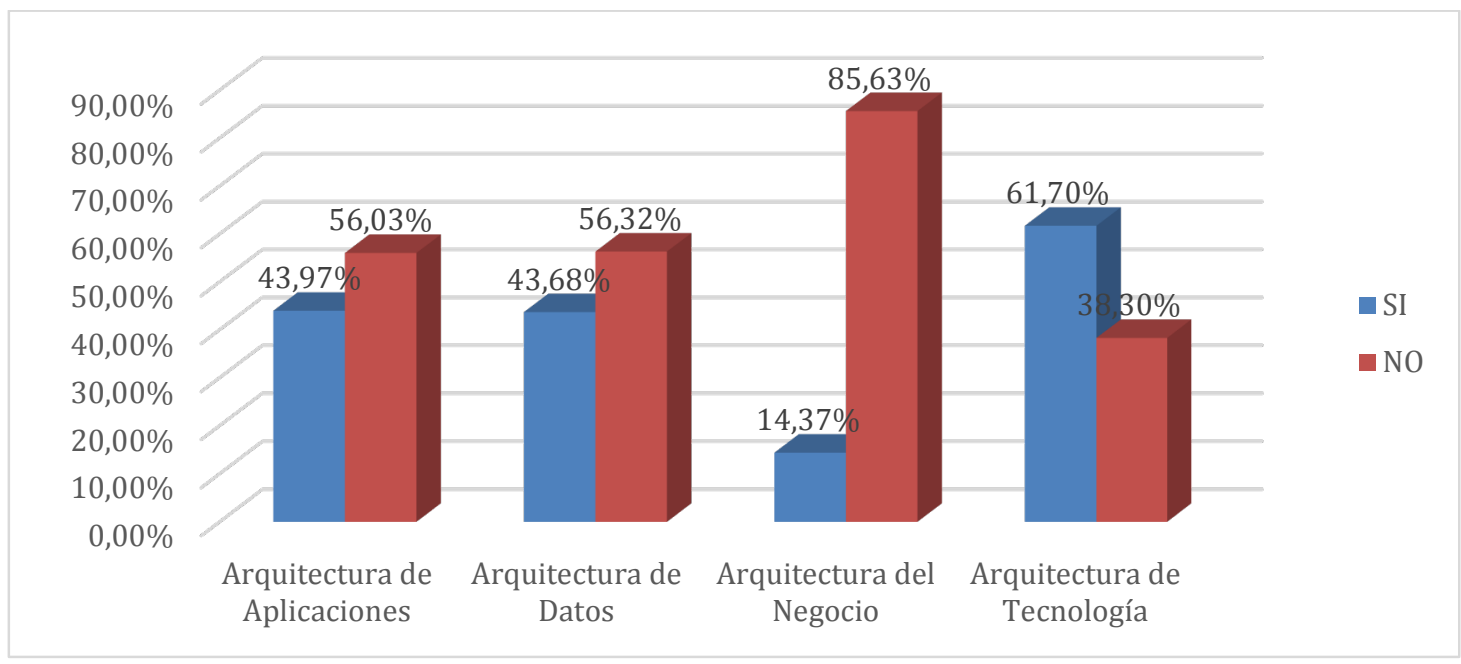

Gráfico 3. Dimensiones de TOGAF, desde la vista de los empleados

Fuente: Elaboración propia

Observamos que la historia se repite teniendo problemas en la Arquitectura de Negocio desde el punto de vista de los empleados. Con lo ya observado en el Gráfico 2 y 3, vamos a contestar la primera pregunta de nuestra investigación. ¿Cuál es el error más común en las microempresas de la ciudad de Cuenca? El error más común en las microempresas de la ciudad de Cuenca se presenta en la Arquitectura del Negocio. Seguida por la Arquitectura de Datos. En tercer lugar, tenemos la Arquitectura de Aplicaciones. Y finalmente tenemos la Arquitectura de Tecnología.

Para lograr que tecnología apoye al negocio, en la consecución de sus objetivos, se requiere tener muy claro hacia dónde va la microempresa. Pero como podemos observar esta es una falencia grande que estas comparten. A continuación, en el Gráfico 4 veremos la Arquitectura de Tecnología desde la perspectiva de los Gerentes Propietarios. 
Dom. Cien., ISSN: 2477-8818

Vol. 5, núm. 3, julio 2019, pp. 487-512

Análisis de la arquitectura empresarial como oportunidad de mejora en las microempresas de la ciudad de Cuenca

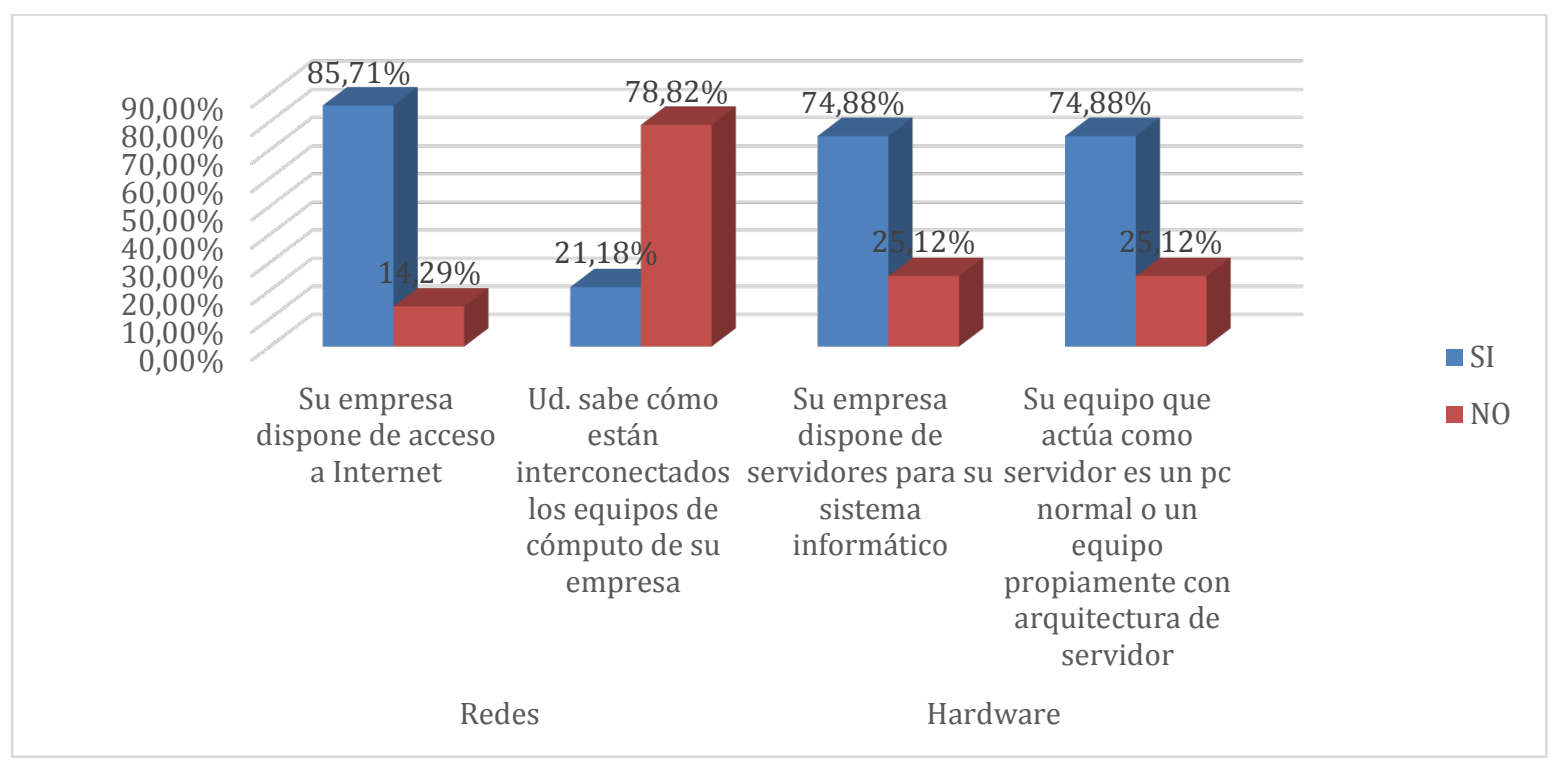

Gráfico 4. Arquitectura de tecnología, desde la vista del Gerente Propietario.

Fuente: Elaboración propia

Aquí podemos notar que, desde la visión del Gerente Propietario, las microempresas tienen un nivel de tecnología básico satisfactorio. Mientras que en el Gráfico 5 observaremos la Arquitectura de Tecnología desde la perspectiva de los empleados. 


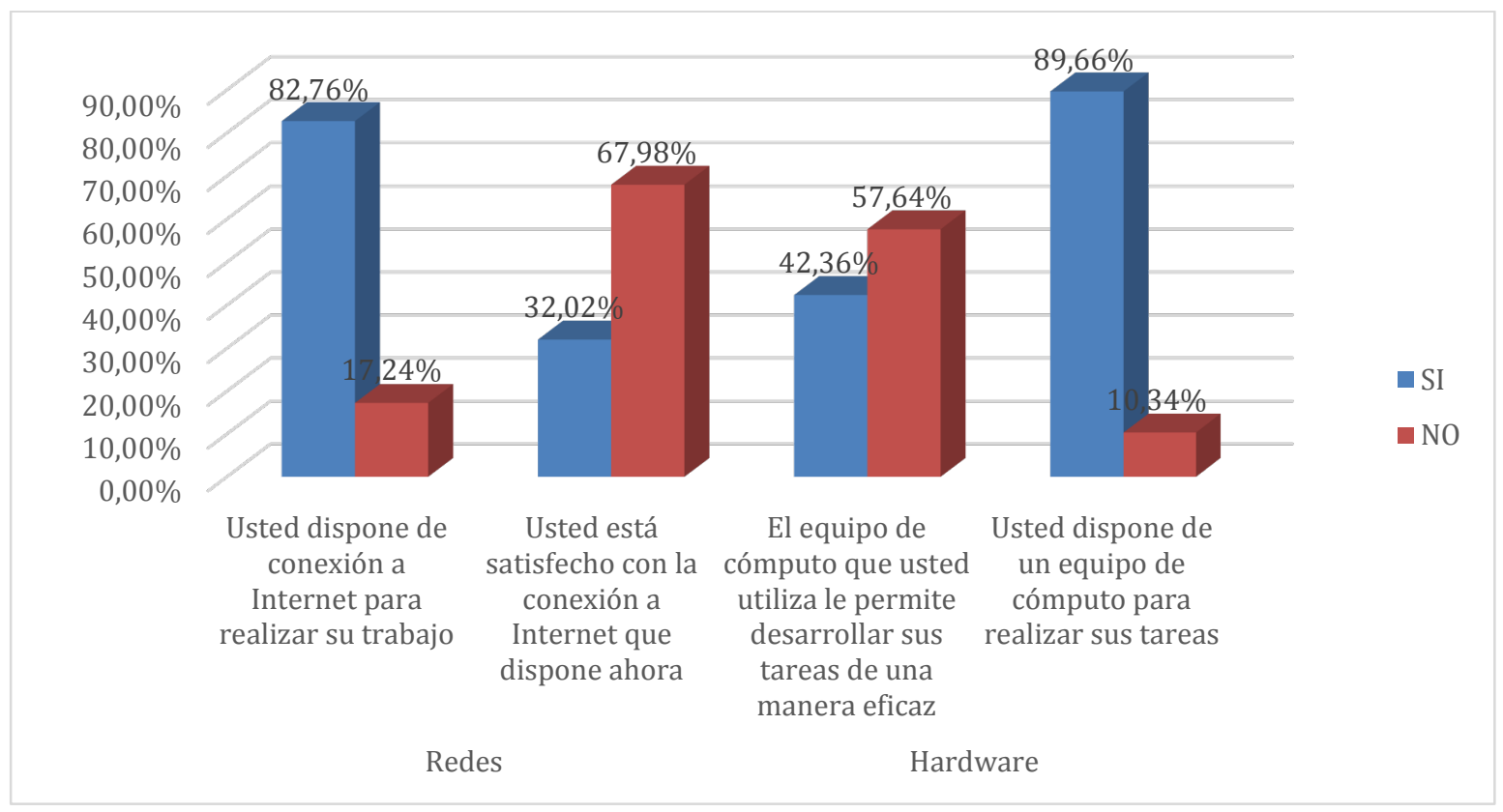

Gráfico 5. Arquitectura de tecnología, desde la vista del empleado

Fuente: Elaboración propia

Desde la vista de los empleados, el nivel tecnológico de la microempresa deja mucho que desear en cuanto a Hardware y a Comunicaciones. Analizando los gráficos anteriores podemos decir que, desde la vista del Gerente Propietario, la microempresa tiene lo necesario para trabajar. Y el Gerente Propietario no ve la necesidad de mejorar o contratar servicios adicionales. Desde el lado del empleado se visualizan falencias como por ejemplo la efectividad de los equipos de cómputo y del internet. Principalmente como resultado del intento de ahorro al adquirir dichos activos, o al contratar servicios de tecnología. Producto del desconocimiento de que Arquitectura de Negocio van a necesitar apoyar con esta tecnología. Con estos resultados hemos contestado la pregunta de investigación: ¿Cuál es el nivel tecnológico de las microempresas de la ciudad de Cuenca?

\section{Elaboración de la propuesta - Visión de Arquitectura}

Como se pudo observar la Arquitectura de Negocio es la que mayores problemas representa en las microempresas. Esto se traduce en un grave problema para el desarrollo de las microempresas. Ya que, si no tenemos un Alineamiento Estratégico, Procesos de Negocio (BPM) o la Gestión de Proyectos definidos correctamente es imposible que la microempresa sobreviva a los cambios de mercado. Esto principalmente, porque al carecer de lo descrito anteriormente, la microempresa no 
tiene el horizonte claro. Lo que puede generar que ante cualquier imprevisto en el mercado la microempresa perezca. Es por esto que esta investigación apunta a poder desarrollar una primera Arquitectura de Negocio.

En el Gráfico 6 se observan los indicadores de la Arquitectura de Negocio desde el punto de vista del Gerente Propietario. Y como observaremos los indicadores con peor puntuación son los que se refieren a la Arquitectura de Negocio. Esto plantea la necesidad de poder definir una primera visión de dicha Arquitectura.

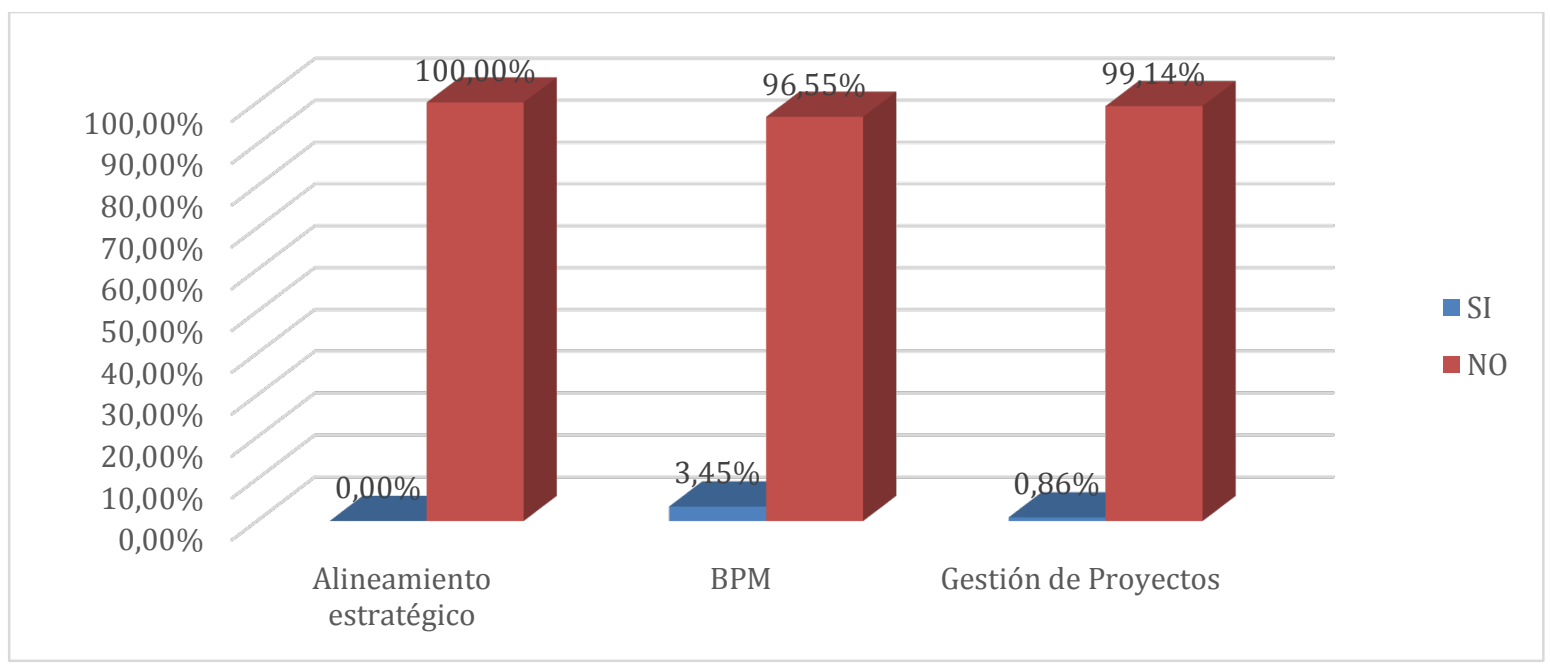

Gráfico 6. Indicadores de la Arquitectura de Negocio, desde la vista de los Gerentes Propietarios

Fuente: Elaboración propia

En el Gráfico 7 tenemos los indicadores de la Arquitectura de Negocio desde el punto de vista de los empleados. Estos indicadores nos permiten ver claramente que las microempresas no tienen una Arquitectura de Negocio. Esto hace que se les dificulte alinear estratégicamente la tecnología para apoyar que la microempresa cumpla sus objetivos. 


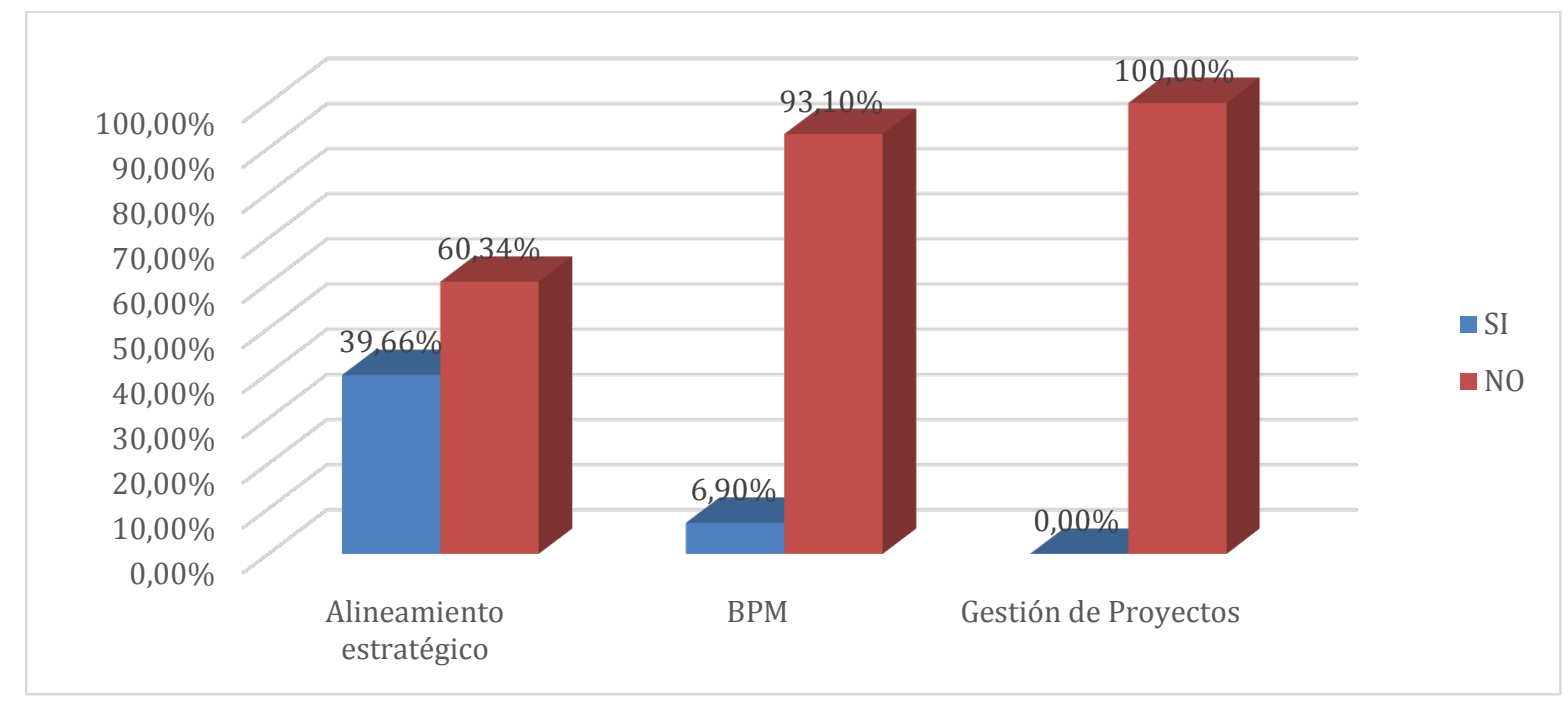

Gráfico 7. Indicadores de la Arquitectura de Negocio, desde la vista de los empleados

Fuente: Elaboración propia

Como resultado de esta investigación podemos plantear un portafolio de proyectos. Portafolio que permita a las microempresas encaminarse y tener claros sus objetivos. Y va a permitirles definir que Arquitectura de Aplicaciones, Arquitectura de Datos y que Arquitectura Tecnológica van a apoyar a las microempresas en su camino a la meta planteada.

\section{Portafolio de Proyectos.}

En la Tabla 3 vamos a observar los problemas encontrados en la Arquitectura de Negocio.

\begin{tabular}{|l|c|c|}
\hline \multicolumn{2}{|l|}{ Arquitectura de Negocio } \\
\hline \multicolumn{2}{|l|}{ Alineamiento Estratégico } & SI \\
\hline Ítem & $0,00 \%$ & $100,00 \%$ \\
\hline $\begin{array}{l}\text { Dispone de todas las herramientas informáticas } \\
\text { para optimizar su trabajo }\end{array}$ & $79,31 \%$ & $20,69 \%$ \\
\hline $\begin{array}{l}\text { Las herramientas informáticas le ayudan a } \\
\text { cumplir con su trabajo }\end{array}$ & \\
\hline
\end{tabular}




\begin{tabular}{|c|c|c|}
\hline $\begin{array}{l}\text { Su empresa tiene definido un plan estratégico } \\
\text { de manera formal }\end{array}$ & $0,00 \%$ & $100,00 \%$ \\
\hline $\begin{array}{l}\text { Su empresa tiene formalmente definida su } \\
\text { misión }\end{array}$ & $0,00 \%$ & $100,00 \%$ \\
\hline $\begin{array}{l}\text { Su empresa tiene formalmente definida su } \\
\text { visión }\end{array}$ & $0,00 \%$ & $100,00 \%$ \\
\hline \multicolumn{3}{|l|}{$\mathrm{BPM}$} \\
\hline $\begin{array}{l}\text { Su empresa tiene definidos sus procesos de } \\
\text { manera formal }\end{array}$ & $3,45 \%$ & $96,55 \%$ \\
\hline $\begin{array}{l}\text { Usted tiene un manual de funciones de su } \\
\text { puesto }\end{array}$ & $6,90 \%$ & $93,10 \%$ \\
\hline \multicolumn{3}{|l|}{ Gestión de Proyectos } \\
\hline Sus proyectos constan de un estudio de riesgos & $0,00 \%$ & $100,00 \%$ \\
\hline $\begin{array}{l}\text { Sus proyectos cuenta con estudios de costo- } \\
\text { beneficio }\end{array}$ & $0,00 \%$ & $100,00 \%$ \\
\hline $\begin{array}{l}\text { Tiene alguna manera para realizar el } \\
\text { seguimiento de sus proyectos en la empresa }\end{array}$ & $0,00 \%$ & $100,00 \%$ \\
\hline $\begin{array}{l}\text { Tiene definida una metodología para } \\
\text { emprender proyectos }\end{array}$ & $3,45 \%$ & $96,55 \%$ \\
\hline $\begin{array}{l}\text { Usted utiliza alguna metodología formal para } \\
\text { manejar sus proyectos en su área }\end{array}$ & $0,00 \%$ & $100,00 \%$ \\
\hline
\end{tabular}

Tabla 3: Ítems de la Arquitectura de Negocio

Fuente: Elaboración propia 
En la Tabla 3 los resultados muestran los problemas de la Arquitectura de Negocio en las microempresas. Mientras que en la Tabla 4 vamos a definir un portafolio de proyectos que permitan generar una primera Visión de Arquitectura de Negocio.

\begin{tabular}{|c|c|}
\hline \multicolumn{2}{|l|}{ Portafolio de Proyectos } \\
\hline Proyecto & Ítem que soluciona \\
\hline \multicolumn{2}{|l|}{ Alineamiento Estratégico } \\
\hline \multirow[t]{3}{*}{$\begin{array}{l}\text { Planteamiento de la misión, visión y un } \\
\text { plan estratégico para la microempresa }\end{array}$} & $\begin{array}{l}\mathrm{Su} \text { empresa tiene definido un plan } \\
\text { estratégico de manera formal }\end{array}$ \\
\hline & $\begin{array}{l}\text { Su empresa tiene formalmente definida } \\
\text { su misión }\end{array}$ \\
\hline & $\begin{array}{l}\text { Su empresa tiene formalmente definida } \\
\text { su visión }\end{array}$ \\
\hline \multirow[t]{2}{*}{$\begin{array}{l}\text { Planteamiento de un Plan Operativo } \\
\text { Anual (POA) }\end{array}$} & $\begin{array}{l}\text { Dispone de todas las herramientas } \\
\text { informáticas para optimizar su trabajo }\end{array}$ \\
\hline & $\begin{array}{l}\text { Las herramientas informáticas le ayudan } \\
\text { a cumplir con su trabajo }\end{array}$ \\
\hline \multicolumn{2}{|l|}{ BPM } \\
\hline $\begin{array}{l}\text { Levantamiento de procesos de negocio de } \\
\text { la microempresa }\end{array}$ & $\begin{array}{l}\text { Su empresa tiene definidos sus procesos } \\
\text { de manera formal }\end{array}$ \\
\hline $\begin{array}{l}\text { Creación de manuales de funciones de los } \\
\text { diferentes departamentos que conformen } \\
\text { la microempresa }\end{array}$ & $\begin{array}{l}\text { Usted tiene un manual de funciones de su } \\
\text { puesto }\end{array}$ \\
\hline Gestión de Proyectos & \\
\hline
\end{tabular}


Análisis de la arquitectura empresarial como oportunidad de mejora en las microempresas de la ciudad de Cuenca

\begin{tabular}{|l|l|}
\hline $\begin{array}{l}\text { Plantear una metodología básica para } \\
\text { ejecución y control de proyectos. }\end{array}$ & $\begin{array}{l}\text { Tiene alguna manera para realizar el } \\
\text { seguimiento de sus proyectos en la } \\
\text { empresa }\end{array}$ \\
\cline { 2 - 2 } & $\begin{array}{l}\text { Tiene definida una metodología para } \\
\text { emprender proyectos }\end{array}$ \\
\cline { 2 - 2 } & $\begin{array}{l}\text { Usted utiliza alguna metodología formal } \\
\text { para manejar sus proyectos en su área }\end{array}$ \\
\hline Plantear una metodología básica de & Sus proyectos cuenta con estudios de \\
análisis de costo-beneficio & costo-beneficio \\
\hline Plantear una metodología básica para el & Sus proyectos constan de un estudio de \\
análisis y mitigación de riesgos & riesgos \\
\hline
\end{tabular}

Tabla 4: Portafolio de proyectos

Fuente: Elaboración propia

La Tabla 4 plantea un Portafolio de Proyectos como oportunidad de mejora para las microempresas comercializadoras de productos para la construcción, de la ciudad de Cuenca. Las Tecnologías de la Información buscan apoyar a que las microempresas logren sus objetivos. Pero si estas no los tienen definidos, el primer paso sería ese. Luego en base a esta Arquitectura del Negocio planteada poder articular las demás dimensiones de Arquitectura Empresarial como son la de Aplicaciones, Datos y Tecnología. 


\section{Conclusiones}

Los Gerentes Propietarios no ven la necesidad de invertir en tecnología. Debido a la falta de una Arquitectura de Negocio que le permita visualizar como la inversión en tecnología va a hacer más rentable su microempresa.

La falta de documentación en la Arquitectura Tecnológica se debe principalmente a que los Gerentes Propietarios no saben que deben exigir de las empresas de outsourcing de TI. Esto se traduce en la dependencia del proveedor de TI ya que es el único que conoce la situación actual de la parte tecnológica de la microempresa.

Al disponer de una Arquitectura de Negocio no solo la tecnología va a apoyar la gestión de la empresa. Si no cada uno de los empleados va a saber qué es lo que la empresa necesita de ellos para alcanzar sus objetivos.

Con la implantación de un Plan Operativo Anual (POA) por área de la microempresa vamos a tener un trabajo organizado. Cada área va a saber en que debe enfocar sus esfuerzos para que la empresa logre sus objetivos.

Una vez implementada una correcta Arquitectura de Negocio se podría comenzar a desarrollar una Arquitectura de Aplicaciones. Que nos entregue información oportuna, mediante una Arquitectura de Datos, para la toma de decisiones. Asentada sobre una Arquitectura de Tecnología adecuada, que apoye a la microempresa a alcanzar sus metas propuestas. Ahorrando recursos ya que solo se adquiriría lo que realmente se necesite para soportar las Arquitecturas ya definidas. Y dando una guía para que el Gerente Propietario sepa que Acuerdos de Nivel de Servicio (SLAs) mínimos debe exigir de sus proveedores de TI. Permitiendo a la microempresa ser más competitiva y adaptarse al cambio. 


\section{Referencias Bibliográficas}

Base, E. N., La, A. E. D. E., Mauricio, R., Salvador, A., Gonzalo, F., Logacho, L., \& Parra, D. M. (2014). Análisis de brecha de arquitectura empresarial, en base a estándares de la industria de telecomunicaciones. Artículos Científicos - Maestría En Gerencia de Sistemas.

Camacho. (2008). Los múltiples retos de las pymes en América Latina. Retrieved October 21, 2018, from

http://noticias.universia.net.co/movilidadacademica/noticia/2008/06/24/242106/multiples-retos-pymes-america-latina.html

Canabal, R., Cabarcas, A., \& Martelo, R. J. (2017). Aplicación de un Esquema de Arquitectura Empresarial (TOGAF) para una Pequeña Empresa (PYME) utilizando Aplicaciones Colaborativas de Google. Informacion Tecnologica, 28(4), 85-92. https://doi.org/10.4067/S0718-07642017000400011

Cardozo, E. (2004). Caracterización de la micro, pequeña y mediana empresa. Guayana.

Censos, I. N. de E. y. (2016). Directorios De Empresas Y Establecimientos, 2016, 68. Retrieved from http://www.ecuadorencifras.gob.ec/documentos/webinec/Estadisticas_Economicas/DirectorioEmpresas/Directorio_Empresas_2016/Principales_ Resultados_DIEE_2016.pdf

Cortés, G. (2013). ASIS - Arquitectura Empresarial e ITIL.

Erika María González Escobar. (2010). FRAMEWORKS DE ARQUITECTURA EMPRESARIAL, DIFERENCIAS ENTRE VERSIONES Y AUTORES. Retrieved June 5, 2018, from https://arquitecturaempresarialcali.wordpress.com/ensayos/togafzachman/

Instituto Nacional de Estadística y Censos. (2017). Ecuador registró 843.745 empresas en 2016. Retrieved September 22, 2018, from http://www.ecuadorencifras.gob.ec/ecuador-registro843-745-empresas-en-2016/ 
Jones, J. (n.d.). El Outsourcing como herramienta de eficiencia en la actividad de desarrollo de software. $\quad$ Retrieved from http://redi.ufasta.edu.ar:8080/xmlui/bitstream/handle/123456789/1581/2017_C_001.pdf?seq uence $=1$

Sandoval, F., Galvez, V., \& Moscoso, O. (2017). Desarrollo de Arquitectura Empresarial usando un Framework con Enfoque Agil ( Development of Enterprise Architecture using a Framework with Agile Approach ). Enfoqute, Vol 8, Iss 1, Pp 135-147 (2017) VO - 8, (1), 135-147. Retrieved from http://proxy1.ncu.edu/login?url=http://search.ebscohost.com/login.aspx?direct=true $\& \mathrm{db}=\mathrm{eds}$ doj\&AN=edsdoj.9604e0a1a2c6441 eaee6cc4cc0f3c664\&site=eds-live

Servicio de Rentas Internas. (2013). Resoluci_n No. NAC-DGERCGC13-00236, publicada en R.O. 956 de 17-05-2013.pdf.

Servicio de Rentas Internas. (2014). Que conforme al articulo 226 de la ConstituciOn de la Republica del Ecuador, las instituciones del Estado, sus organismos ', dependencias, las servidoras , o servidores pablicos y las personas que actUen en virtud de una potestad estatal, ejerceran so.

The Open Group. (2011). Guia de bolsillo TOGAF 9.1. Retrieved from https://www.vanharen.net/Samplefiles/9789087537104SMPL.pdf

The Open Group. (2012). TOGAF The Open Group Architecture Framework. Retrieved June 5, 2018, from https://arquitecturaempresarialcali.wordpress.com/ea-frameworks/togaf/

Torres, M., Paz, K., \& Salazar, F. (2006). TAMAÑO DE UNA MUESTRA PARA UNA INVESTIGACIÓN DE MERCADO. Boletín Electrónico, 2, 1-13. 